\title{
Aderência dos orçamentos dos estados brasileiros à luz da teoria do equilíbrio pontuado
}

\section{Marlon Coqueiro Galdino}

\section{Maria Elisabeth Moreira Carvalho Andrade}

Universidade Federal de Uberlândia (UFU), Uberlândia, MG - Brasil

Este artigo tem como objetivo analisar o comportamento do orçamento dos estados brasileiros. $\mathrm{O}$ intuito da análise foi verificar a aderência dos orçamentos dos governos estaduais à luz da teoria do equilíbrio pontuado. A análise constituiu-se a partir dos dados orçamentários dos estados e do Distrito Federal compreendendo os anos de 2002 até 2018. Foram selecionadas ainda quatro funções de despesas para a análise, além do orçamento geral. Testou-se a normalidade dos dados para demonstrar o incrementalismo e a curtose para verificar as pontuações no orçamento. Observou-se que, no caso do orçamento geral, vinte e cinco estados apresentaram-se com modelo incremental. Quanto às despesas por função, foi verificado que somente os Estados do Espírito Santo e do Rio de Janeiro mostraram evidências da presença do equilíbrio pontuado para as funções de saúde e segurança pública, respectivamente. Por fim, concluiu-se que a teoria do equilíbrio pontuado não se aplica, de modo geral, aos orçamentos dos estados do Brasil, salvo algumas exceções. Portanto, a hipótese da pesquisa de que o orçamento segue uma tendência regular, pontualmente alterada por grandes variações, não foi confirmada.

Palavras-chave: teoria do equilíbrio pontuado, incrementalismo, orçamento público 


\section{Adherencia de los presupuestos de los estados brasileños a la luz de la teoría del equilibrio puntuado}

Este artículo tiene como objetivo analizar el comportamiento del presupuesto de los estados brasileños. El propósito del análisis era comprobar la adherencia de los presupuestos de los gobiernos estatales y Distrito Federal a la luz de la teoría del equilibrio puntuado. El análisis se basó en los datos de los presupuestos de los estados para los años 2002 a 2018. También se seleccionaron cuatro funciones de gasto para el análisis, además del presupuesto general. La normalidad de los datos fue probada para demostrar el incremento y la curtosis para verificar puntajes del presupuesto. Se observó que, en el caso del Presupuesto General, veinticinco estados se presentaron con un modelo incremental. Con respecto a los gastos por función, se encontró que solo los estados de Espírito Santo y Río de Janeiro mostraron evidencia de la presencia del Equilibrio Puntuado para las funciones de salud y seguridad pública, respectivamente. Finalmente, se concluyó que la teoría del Equilibrio Puntuado no se aplica, en general, a los presupuestos de los estados de Brasil, con algunas excepciones. Por tanto, la hipótesis de la investigación no ha sido confirmada. No se ha confirmado la investigación de que el presupuesto sigue una tendencia regular, ocasionalmente alterada por grandes variaciones.

Palabras clave: teoría del Equilibrio Puntuado, el incremento, presupuesto público

\section{Adherence of brazilian states' budgets in the light of the punctuated equilibrium theory}

This article aims to analyze the behavior of the budget of the Brazilian states. The purpose of the analysis was to verify the adherence of the budgets of the state governments in the light of the Theory of Punctuated Equilibrium. The analysis was based on the budgets data of the states and Distrito Federal covering the years 2002 to 2018. Four expenditure functions were also selected for analysis, in addition to the general budget. Normality of the data was tested to demonstrate incrementalism and kurtosis to verify budget punctuations. It was observed that in the case of the General Budget, twenty-five states presented themselves with an incremental model. Regarding expenses by function, it was found that only the states of Espírito Santo and Rio de Janeiro showed evidence of the presence of the Punctuated Equilibrium for the Health and Public Safety functions, respectively. Finally, it was concluded that the Punctuated Equilibrium Theory does not apply, in general, to the budgets of the states of Brazil, with some exceptions. Therefore, the research hypothesis has not been confirmed. the research that the budget follows a regular trend, occasionally altered by large variations, has not been confirmed.

Keywords: punctuated equilibrium theory, incrementalism, public budget 


\section{Introdução}

O orçamento é uma importante ferramenta de gestão. Por meio dele é possível saber os recursos disponíveis e onde devem ser aplicados. Em termos formais, pode-se dizer que o orçamento é um instrumento de planejamento em que se confrontam as receitas com as despesas (GIACOMONI, 2017). Os estudos sobre orçamentos modernos surgiram no fim da década de 1950 para o início da década de 1960, com as pesquisas de Lindbloom (1959) e Wildavsky (1961), em que passam a ser tratados como um instrumento de administração (GIACOMONI, 2017).

Os estudos que Wildavsky (1961) difundiram uma nova uma teoria de como seria o modelo orçamentário utilizado nos Estados Unidos: a teoria do incrementalismo, a qual diz que os orçamentos são feitos com base no ano anterior, em que são os mesmos sofrendo pequenas variações, seja em algum tipo de despesa específica ou no orçamento geral. A partir do estudo de Wildavsky (1961) é que Baumgartner e Jones (1991) desenvolveram uma nova teoria para complementar a teoria incremental, denominada teoria do equilíbrio pontuado (TEP), em que afirmam que os orçamentos são incrementais, mas que sofrem grandes rupturas em algum período.

Apesar da teoria incremental ser a mais utilizada nos estudos que tratam sobre o orçamento no Brasil (BARCELOS, 2012), a teoria do equilíbrio pontuado (TEP) tem espaço para ser uma alternativa à teoria incremental, visto que ainda foi pouco utilizada nos estudos sobre orçamento no Brasil. E pela realidade que o país vive, com constantes crises econômicas e políticas ao longo do tempo, talvez seja uma teoria mais aplicável à realidade brasileira, podendo ela ser de grande ajuda para os pesquisadores da área de orçamento, contribuindo para uma nova visão sobre os orçamentos, seja no âmbito da administração pública, quanto na administração privada.

Outro aspecto que torna a discussão ainda mais relevante é quanto à competição fiscal que pode existir entre os estados (TIEBOUT, 1956). Porsse, Haddad e Ribeiro (2008) alertam que, no Brasil, a prática de incentivos fiscais é comum, em que o intuito é reter ou atrair investimentos, mas que geralmente não são avaliados os custos dessas renúncias 
nem os seus benefícios. Essa competição pode gerar benefícios, obtendo novas receitas e aumentando o PIB, como também pode apresentar resultados ruins (NASCIMENTO, 2008).

No Brasil, os orçamentos públicos federais já foram pesquisados, e observados quais os tipos de modelos utilizados. Fittipaldi, Costa e Araújo (2017) verificaram, sob a ótica da teoria incremental, que, entre os anos de 1995 e 2014 os orçamentos da União seguiram o perfil do incrementalismo em que pouco havia assimetria entre os gastos estatais.

Silvestre e Araújo (2015) pesquisaram sobre os governos municipais do Ceará para a verificação se a TEP poderia ser aplicada nos municípios daquele estado. Foi verificado que os investimentos públicos seguem uma estabilidade durante um tempo, mas que sofrem rupturas em algum momento, o que eles concluíram satisfazer aos pressupostos da TEP.

Devido ao pequeno número de estudos acerca dos orçamentos estaduais e da teoria do equilíbrio pontuado no Brasil, estabelece-se a pergunta da pesquisa: qual o comportamento da execução orçamentária dos estados brasileiros à luz da teoria do equilíbrio pontuado? Em que se tem como objetivo verificar a aderência dos orçamentos dos governos estaduais em relação à teoria do equilíbrio pontuado. E que tem como hipótese de pesquisa que o orçamento segue uma tendência regular, pontualmente alterada por grandes variações.

Esta pesquisa se faz relevante devido à utilização de uma literatura ainda não muito utilizada no Brasil: a teoria do equilíbrio pontuado. Essa literatura é utilizada com certa frequência no ramo de políticas públicas e pouco usual ainda nos estudos sobre orçamento. Portanto, esta pesquisa contribui para preencher a lacuna na literatura nacional no que se refere à teoria do equilíbrio pontuado.

A teoria do equilíbrio pontuado se faz relevante ao considerar o contexto do país em meio às crises e mudanças de políticas públicas ao longo do tempo. Deste modo, entende-se que a teoria do equilíbrio pontuado seja a teoria mais aplicável, podendo ela ser de grande valia para os pesquisadores da área de orçamento, contribuindo para uma nova visão sobre os orçamentos, seja no âmbito da administração pública, quanto na administração privada. 


\section{Referencial teórico}

\subsection{Teorias dos Modelos Orçamentários}

Diversos modelos teóricos são utilizados quando se trata de agenda pública. No âmbito do orçamento existem quatro modelos que são frequentemente utilizados: julgamento serial, incrementalismo, múltiplos fluxos e equilíbrio pontuado.

Wildavsky (1961) havia identificado que grande parte da literatura da época estava preocupada em uma reforma neutra e que beneficiasse a todos. Uma carência que se apresentava à época era a inexistência de uma teoria orçamentária. Segundo ele, uma teoria orçamentária normativa é mais do que um exercício acadêmico, ela deve guiar efetivamente a tomada de decisões governamentais (DAVIS; DEMPSTER; WILDAVSKY, 1966).

O modelo incremental foi uma proposta desenvolvida por Wildavsky, em 1961, tendo em vista a necessidade de se fazer reformas nos orçamentos americanos. O modelo incremental ganhou aspecto de teoria por um estudo realizado por Davis, Dempster e Wildasvsky (1966) a respeito dos orçamentos do governo federal americano, em um período que compreendeu os anos de 1943 a 1966. Os autores encontraram que os orçamentos de um ano para outro pouco variavam, sofrendo somente incrementos. Para o Wildasvsky (1961), o aspecto crucial do orçamento é quais preferências devem prevalecer nas disputas sobre quais atividades devem ser realizadas e em que grau, sob a perspectiva de recursos limitados.

Williamson (1967) fez ressalvas em relação ao trabalho desenvolvido por Davis, Dempster e Wildavsky, visto que, para ele, a teoria do modelo incremental não apresentava todos os pressupostos comportamentais e que se trata de um modelo sem valor preditivo. Apesar disso, Williamson (1967) classificou o trabalho de Davis, Dempster e Wildavsky como uma importante contribuição para o desenvolvimento da literatura acerca da distribuição.

Outras críticas feitas ao trabalho de Davis, Dempster e Wildavsky foram realizadas por Wanat (1974), que tinha a opinião de que para ser tratado como racionalidade limitada, o incrementalismo teria que apresentar duas formas essenciais: uma é a 
descritiva, em que se deve mostrar mudanças no orçamento, e a segunda é a explicativa que, como o próprio nome diz, busca explicar a razão dessas mudanças. Para Wanat (1974), o incrementalismo só apresentava a sua forma descritiva, sem explicar de fato a razão de ter acontecido tais mudanças.

Em 1976, Allen Shick afirmou que a teoria incremental, desenvolvida por Wildavsky, estava sendo revisada, pois o modelo do orçamento americano havia mudado nos Estados Unidos devido ao mau desempenho da economia americana na década de 1960 e início da década de 1970. Shick (1976) propunha como primeiro passo a verificação da escassez juntamente com o orçamento. Escassez de recurso pode apresentar orçamento diferente.

Como alternativa à teoria incremental, Padgett (1980) desenvolveu uma nova teoria chamada julgamento serial, a qual diz que os orçamentos seguem padrões, tendo um aumento marginal em suas bases, mas que também ocorrem as mudanças nos orçamentos, que podem ser radicais e catastróficas, e que essas mudanças são normais para a rotina do governo federal. Tal teoria tem como base a racionalidade limitada (CARVALHO, 2017), da mesma forma que a teoria trabalhada por Wildavsky, Davis e Dempster (1966).

A teoria do julgamento serial, segundo Padgett (1980), apresenta uma maior flexibilidade temporal em relação ao modelo incremental. O próprio nome dessa teoria já mostra as duas principais diferenças em relação ao incrementalismo: primeiro que é realizada uma pesquisa sequenciada e ordenada de orçamentos, criando assim as séries; e segundo que a decisão é tomada por meio de uma seleção não determinística baseada na aplicação ligeiramente ambígua de "julgamento informado", isto é, o direcionamento da pesquisa a ser tomada é feito pelo tomador de decisão, o que implica na racionalidade limitada e nas semelhanças com o modelo incremental (PADGETT, 1980).

Ollaik et al.(2012) afirmam que, apesar do modelo de julgamento serial ser parecido com o modelo incremental, há diferenças. Mesmo ambos utilizando uma base fixa de referência, o julgamento serial tem como principal ponto que a seleção final não é determinística, isto é, o gestor ou tomador de decisão escolhe a atitude que quer tomar com base em informações (julgamentos informados), considerando méritos políticos e fiscais, então definindo se é melhor aumentar ou diminuir os níveis orçamentários para determinadas funções (OLLAIK et al. 2012) 
O modelo de múltiplos fluxos foi desenvolvido por Kingdon em 1984, em que se afirma que existem três fluxos que são apresentados de forma separada e independentes: os fluxos de problemas, os fluxos políticos e os fluxos de políticas. O primeiro consiste naqueles problemas que os cidadãos querem que sejam tratados (ZAHARIADS, 2007 apud Nowlin, 2011); o fluxo político é aquele que pode incluir a opinião pública e o controle partidário; e, por fim, o fluxo de políticas, que consiste nas ideias e soluções desenvolvidas por especialistas que esperam que estas sejam implementadas (NoWLIN, 2011).

A convergência dos três fluxos, para Metten et al. (2015), abre a chamada "janela de oportunidade política" no processo de definição da política, o que cria a demanda para adição de um novo item. Doeser e Eidenfaçk (2013) apontam que um dos conceitos mais abordados para "janela de oportunidade" refere-se ao momento que ocorre algum tipo de mudança estrutural em que é criado um momento de oportunidade em que o tomador de decisão (líder) apresente novas políticas públicas. O conceito utilizado quanto às políticas públicas diz que as janelas de oportunidades são os períodos de maior receptividade dos atores políticos, em que defensores têm mais poder para apresentar uma nova proposta ou mesmo para pressionar a solução de um problema (KINGDON, 1984 apud NUGENT; SAURUGGER, 2002).

Ridde (2009) realizou um estudo para expandir os conceitos da teoria de múltiplos fluxos além dos países desenvolvidos para verificar se em países de baixa renda essa teoria era aplicável. Foi feita uma avaliação sobre a saúde pública e foi constatado que a teoria era aplicável e que havia uma falha no processo dos fluxos de políticas (implementação). Travis e Zahariadis (2002) verificaram que as hipóteses contidas na Teoria de Múltiplos Fluxos também expandiram os estudos ao tratarem das políticas externas, verificando-se que as expectativas teóricas presentes na teoria também se confirmam nesses casos.

$E$, por fim, tem-se uma teoria mais recente, também como alternativa ao incrementalismo. Baumgartner e Jones (1991) desenvolveram uma outra teoria como complemento à teoria incremental, que é a teoria do equilíbrio pontuado, que consiste no fato de que os entendimentos do poder público podem mudar ao longo do tempo. Defendida por Baumgartner e Jones (1991), a teoria sustenta-se em que os orçamentos são incrementais e sofrem rupturas ao longo do tempo, ou seja, o orçamento sofre pequenas mudanças ano a ano e em determinado momento sofre abrupta mudança em alguma área específica. 
Baumgartner e Jones (1991) apresentam três etapas de como ocorrem as mudanças: a primeira é a atribuição inicial sobre a questão a um local e que esta pode estar associada a uma atenção positiva; a segunda etapa é quando o conflito não se expande para todos os locais, sendo que algumas áreas terão somente pequenas mudanças; por fim, a terceira etapa é que à medida que o conflito se expande, a mudança de local e imagem será mais brusca e simultânea.

Jones e Baumgartner (2005) alertaram que o modelo do equilíbrio pontuado desenvolvido em 1993, diferentemente do incremental, não aparentava um modelo com valor preditivo, ou seja, ele não servia como algo para escolhas políticas, mas sim para explicar mudanças nos subsistemas encontrados nos orçamentos. Entretanto, eles reconhecem que, muitas vezes, o equilíbrio pontuado é tratado como uma alternativa para o incrementalismo nas escolhas públicas.

Apesar da presença e utilização dos quatro modelos, todos eles têm suas limitações. Sobre as falhas dos modelos, Ollaik et al. (2012) afirmam que: o modelo incremental não explica mudanças bruscas; que o julgamento serial tem uma difícil aplicação empírica; que os fluxos múltiplos têm sua previsibilidade limitada e os fluxos não são independentes; e que o equilíbrio pontuado também apresenta uma previsibilidade limitada e também possui difícil aplicação empírica.

\subsection{Estudos correlatos}

Os estudos utilizando a teoria do equilíbrio pontuado têm aumentado ao longo dos anos. Os primeiros estudos de Baumgartner e Jones (1991) levantavam a teoria em relação aos orçamentos do governo federal dos Estados Unidos da América, não contendo ainda níveis mais baixos dos entes federativos.

Jordan (2003) desenvolve um estudo tratando da teoria em relação aos governos locais estadunidenses, isto é, aos municípios. Nesse estudo, Jordan (2003) busca determinar as funções em que ocorrem as pontuações e a probabilidade para que ocorram futuras rupturas. A autora concluiu que funções alocacionais (bombeiro, polícia e saneamento) seguem uma estabilidade, diferentemente das não-alocacionais (parques e recreação, edifícios públicos e estradas), que apresentam diversas pontuações. 
O estudo de Mortensen (2005) aplica a teoria em um lugar diferente dos Estados Unidos, passando a aplicar o estudo na Dinamarca. Outro ponto de que trata esse estudo é a análise de quatro funções para a identificação de onde existe maior probabilidade de que ocorram grandes variações dos gastos. Concluiu-se que os orçamentos dinamarqueses também sofrem grandes períodos de estabilidade e em algum momento há uma ruptura, comprovando a TEP. Foram ainda verificados que os gastos com biblioteca e estradas apresentam variações mais bruscas que os gastos com escola e cuidados infantis.

Breuning e Koski (2006) analisaram os orçamentos dos cinquenta estados americanos, em um período que compreende 18 anos e dez funções, a fim de descobrir o comportamento dos gastos nos estados. Eles chegaram às conclusões de que as categorias orçamentárias estaduais são interdependentes, geralmente pontuadas e nos mais diversos graus de pontuação, mostrando também que a TEP se aplica também nos casos dos orçamentos estaduais.

No Brasil, os estudos que envolvem a teoria do equilíbrio pontuado ainda são incipientes. Silvestre e Araújo (2015) realizaram um estudo sobre as variações das transferências nos orçamentos dos municípios do Estado do Ceará. Observaram que os municípios dependem das transferências de recursos da União. Um outro resultado que foi apresentado pelos autores é que os investimentos públicos mantêm períodos de estabilidade e logo em seguida ocorrem grandes alterações no orçamento, o que comprova os pressupostos da teoria do equilíbrio pontuado.

Carvalho (2018) pesquisou sobre os orçamentos do Governo Federal, compreendidos entre os anos de 1980 e 2014, para verificar a aderência à teoria do equilíbrio pontuado. $\mathrm{Na}$ análise, observou-se que em alguns anos houve uma maior amplitude das variações nos gastos federais, em especial nos anos de 1986, 1989 e 1990, período que compreende uma grande instabilidade monetária no país, e notou-se que a partir do ano de 1996 houve um maior equilíbrio no orçamento, justificado pelo fato do equilíbrio monetário do Brasil.

Brasil e Capella (2019) desenvolveram um estudo com a finalidade de observar a agenda pública voltada à saúde. Para tanto, eles selecionaram cinco variáveis para a análise das políticas públicas, sendo uma delas o orçamento. Nessa análise sobre o orçamento verificou-se um alto grau de curtose, mostrando que no orçamento federal de saúde há 
pontuações no orçamento. A conclusão do estudo é que ao ser considerada a dinâmica, foram verificadas manutenções nas políticas com ajustes discretos e incrementais na política de saúde durante a maior parte do tempo, mas também foram pontuados momentos de grandes rupturas e transformações ao longo do período (BRASIL; CAPELLA, 2019), o que pode se configurar como teoria do equilíbrio pontuado.

Melo et al (2018) analisaram o processo de decisão em uma instituição de ensino superior (IES), observando dimensõe, como: ambiente, participação, atenção e agenda, dinâmica político institucional e o processamento de informações. Para tanto, os autores utilizaram os seguintes modelos teóricos de orçamento: incremental, julgamento seriado, fluxos múltiplos e equilíbrio pontuado. O resultado obtido da pesquisa é que não é possível afirmar qual modelo é aplicado na instituição, mas foi possível observar que coexiste relação entre os modelos e a tomada de decisão na IES (MELo et al., 2018).

\section{Aspectos metodológicos}

O universo da pesquisa é composto pelos 26 estados do Brasil e o Distrito Federal, totalizando 27 entes. Foram selecionadas quatro funções de despesas no orçamento para verificação da teoria e separadas entre despesas com limites mínimos constitucionais e sem limites constitucionais: saúde e educação para as que contêm limites, e segurança pública e cultura para as sem limites constitucionais. O objetivo dessa separação foi verificar se há diferenças entre a execução daquelas despesas que a CF/88 obriga a gastar um mínimo daquelas despesas em que não há a mesma obrigatoriedade mínima do gasto.

Os valores orçamentários colhidos correspondem aos anos de 2002 até 2018. O período inicial da pesquisa foi escolhido assim por conta da padronização dos dados, pois antes de 2002 diversas funções eram condensadas em uma só, não podendo fazer segregação entre alocação das despesas. E o último ano relativo ao período corresponde ao último ano com dados integralmente disponíveis na data da realização da coleta.

Os dados foram coletados por meio da plataforma Finanças do Brasil (Finbra) geridos pela Secretaria do Tesouro, a qual contém todos os dados financeiros e 
orçamentários dos estados brasileiros. Os valores coletados se referem ao primeiro estágio da despesa, o empenho, pois nesta etapa o governo cria o compromisso em reservar o recurso para o pagamento.

Os valores coletados para a pesquisa foram corrigidos pela inflação a fim de manter a paridade dos dados em relação ao outro ano, isto é, diminuindo o efeito da inflação nos valores orçamentários. Para a devida correção, utilizou-se o Índice de Preços do Consumidor Amplo (IPCA) mensurado pelo Instituto Brasileiro de Geografia e Estatística (IBGE).

Então foi verificado que as variações anuais têm o objetivo de verificar a porcentagem que os valores diminuíram ou aumentaram de um ano para o outro, e o cálculo é realizado de forma simples:

$$
\operatorname{Var}_{\text {Ano1 }}=\left(\mathrm{V}_{\text {Ano1 }}-\mathrm{V}_{\text {Anoo }}\right) / \mathrm{V}_{\text {Anoo }}
$$

Sendo:

$$
\begin{aligned}
& \operatorname{Var}_{\text {Ano1 }} \text { Variação do ano } 1 \\
& \mathrm{~V}_{\text {Ano1 }}=\text { Valor do ano } 1 \\
& \mathrm{~V}_{\text {Anoo }}=\text { Valor do ano } 0
\end{aligned}
$$

Primeiramente, foi feita uma estatística descritiva dos dados, cujo objetivo foi de verificar como é que se comportam os dados, verificar os valores máximos e mínimos observados nos orçamentos dos estados, bem como averiguar o coeficiente e as médias encontradas nos respectivos valores por função de despesas e também no valor do orçamento geral do estado.

A pesquisa foi realizada a partir dos métodos desenvolvidos por Baumgartner e Jones (1991) para a verificação da teoria do equilíbrio pontuado em que são descritos dois tipos de testes: os testes de normalidades - que têm como objetivo verificar a existência de dados não normais - e os testes de assimetria e grau de curtose para a verificação das pontuações no orçamento. 
Cabe ressaltar que, assim como determinam os estudos de Baumgartner e Jones (1991) e também em estudos posteriores como o de Jordan (2003) e Breunig e Koski (2006), os dados foram testados de forma separada, isto é, cada função foi testada por estado de forma isolada das demais, mostrando então os 17 anos que compõem o período do universo da pesquisa.

Foram realizados os testes de normalidade (Shapiro-Wilk) e o teste de curtose nas variações anuais dos orçamentos totais por estado. Os testes, num primeiro momento, foram realizados nos orçamentos totais com o objetivo de saber o comportamento das finanças gerais daquele estado, para, então, no segundo momento, serem realizados os testes por função. Os dados estatísticos foram tratados utilizando o programa STATA, em sua versão 15.

Após a realização da análise descritiva, foram feitos testes de normalidade e testes de curtose. No caso desta última, estabelece-se que o valor para a verificação de uma curva leptocúrtica é referente aos valores acima de 3 . Ou seja, caso a distribuição da variação orçamentária seja normal e o grau de curtose seja maior que 3, então aquele estado apresenta a teoria do equilíbrio pontuado em seu orçamento. Caso a distribuição seja apenas normal, sem a apresentação de curvas leptocúrticas, então aquele estado apresenta-se como um modelo incremental de orçamento, conforme descrito por Jones e Baumgartner (2005), em que dizem que para o orçamento ser incremental, basta que os testes de normalidade de seus dados sejam considerados com distribuição normal.

\section{Resultados e discussão}

A primeira etapa da pesquisa constitui-se na análise geral dos orçamentos, ou seja, a verificação do orçamento total de cada Estado. Na Tabela 1 é possível observar os valores médios das variações, os mínimos, os máximos e os testes de normalidade e de curtose. 
Tabela 1 | Análise das variações no orçamento total

\begin{tabular}{|c|c|c|c|c|c|c|}
\hline \multirow{2}{*}{ Estado } & \multicolumn{3}{|c|}{ Descritiva } & \multicolumn{3}{|c|}{ Normalidade e Curtose } \\
\hline & Média & Mínimo & Máximo & Teste & Valor-p & Curtose \\
\hline $\mathrm{AC}$ & $11,27 \%$ & $-10,82 \%$ & $28,33 \%$ & 0,9470 & 0,4440 & $-0,6766$ \\
\hline$A L$ & $8,84 \%$ & $-3,45 \%$ & $20,35 \%$ & 0,9608 & 0,6764 & $-0,7090$ \\
\hline AM & $10,24 \%$ & $-7,69 \%$ & $23,46 \%$ & 0,9746 & 0,9066 & 0,2152 \\
\hline AP & $10,64 \%$ & $-9,19 \%$ & $28,51 \%$ & 0,9464 & 0,4345 & $-0,6931$ \\
\hline BA & $10,00 \%$ & $3,76 \%$ & $17,59 \%$ & 0,8933 & 0,0629 & $-0,9520$ \\
\hline CE & $9,65 \%$ & $-9,81 \%$ & $32,26 \%$ & 0,9373 & 0,3167 & 0,1411 \\
\hline DF & $8,91 \%$ & $-27,35 \%$ & $45,76 \%$ & 0,9074 & 0,1056 & 1,3445 \\
\hline ES & $8,92 \%$ & $-11,55 \%$ & $23,82 \%$ & 0,9504 & 0,4952 & $-0,1814$ \\
\hline GO & $10,85 \%$ & $-0,28 \%$ & $21,48 \%$ & 0,9118 & 0,1244 & $-1,4562$ \\
\hline MA & $11,82 \%$ & $-10,04 \%$ & $32,19 \%$ & 0,9496 & 0,4837 & 0,5048 \\
\hline MG & $10,11 \%$ & $1,15 \%$ & $21,13 \%$ & 0,9658 & 0,7674 & $-0,6873$ \\
\hline MS & $10,89 \%$ & $-9,31 \%$ & $26,04 \%$ & 0,9597 & 0,6556 & $-0,4287$ \\
\hline MT & $11,61 \%$ & $-3,23 \%$ & $28,27 \%$ & 0,9641 & 0,7363 & $-1,0652$ \\
\hline PA & $12,13 \%$ & $1,74 \%$ & $23,22 \%$ & 0,9491 & 0,4754 & $-0,5915$ \\
\hline PB & $9,15 \%$ & $1,39 \%$ & $19,05 \%$ & 0,9224 & 0,1844 & $-1,2947$ \\
\hline PE & $9,68 \%$ & $-2,91 \%$ & $22,92 \%$ & 0,9614 & 0,6867 & $-1,0231$ \\
\hline PI & $10,99 \%$ & $-0,05 \%$ & $27,23 \%$ & 0,9537 & 0,5510 & $-0,3608$ \\
\hline PR & $11,05 \%$ & $-10,82 \%$ & $40,94 \%$ & 0,7803 & $0,0015^{*}$ & 6,3413 \\
\hline RJ & $7,49 \%$ & $-12,61 \%$ & $19,46 \%$ & 0,8851 & $0,0467 *$ & 1,3039 \\
\hline $\mathrm{RN}$ & $9,05 \%$ & $-7,76 \%$ & $22,33 \%$ & 0,9710 & 0,8551 & $-0,6861$ \\
\hline RO & $10,17 \%$ & $-7,76 \%$ & $26,55 \%$ & 0,9489 & 0,4725 & 1,0309 \\
\hline $\mathrm{RR}$ & $12,79 \%$ & $-34,61 \%$ & 49,19\% & 0,9593 & 0,6490 & 0,5394 \\
\hline RS & $9,99 \%$ & $-6,12 \%$ & $22,15 \%$ & 0,9677 & 0,7993 & 0,6999 \\
\hline SC & $8,44 \%$ & $-11,98 \%$ & $21,63 \%$ & 0,9468 & 0,4410 & 1,6709 \\
\hline SE & $9,56 \%$ & $-7,17 \%$ & $24,46 \%$ & 0,9517 & 0,5166 & $-1,2320$ \\
\hline SP & $9,39 \%$ & $-3,22 \%$ & $25,64 \%$ & 0,9782 & 0,9477 & $-0,1121$ \\
\hline TO & $9,87 \%$ & $-4,09 \%$ & $22,59 \%$ & 0,9708 & 0,8514 & 0,0636 \\
\hline
\end{tabular}

Fonte: resultados da pesquisa *Nível de significância 5\% 
Observa-se que a média de variação entre os estados não muda muito, os níveis das mudanças variam entre $7,49 \%$ e $12,79 \%$, o que já demonstra que os orçamentos são incrementais, o que mostra que não houve mudanças dos métodos usados na década de 1970 aos quais Shick (1976) alertava que aconteciam nos orçamentos estadunidenses, e que por isso o sistema de planejamento, programação e orçamentação não deu certo.

Outro ponto a ser observado é que as variações no orçamento variam tanto para mais quanto para menos. O Distrito Federal (DF), por exemplo, apresenta o maior mínimo e o maior máximo de variação, $27,35 \%$ e $45,76 \%$, respectivamente. Nota-se que, apesar de apresentar valores tão distintos entre o mínimo e o máximo, o DF é apresentado com dados normais ao considerar um nível de significância de $5 \%$, o que pode indicar também que o modelo incremental se confirme.

Apesar da maioria dos estados se apresentarem com dados normais, verifica-se que nos Estados do Paraná (PR) e do Rio de Janeiro (RJ), considerando um nível nominal de significância de 5\%, não houve uma distribuição normal nos orçamentos ao longo dos 16 anos analisados. No caso do Estado do Paraná, o que fez com que os dados se alterassem foram os valores referentes à previdência que quase triplicaram do ano de 2015 para o ano 2016, sem motivos aparentes, podendo ser erro de lançamento na plataforma do governo.

No caso do Rio de Janeiro (RJ), isso se deve ao fato de o estado ter se apresentado nos últimos anos em meio a sua condição financeira distinta. Isso pode ter acontecido devido a alguns fatores que podem ter afetado a receita e a organização do estado, por exemplo, em 2013 houve mudanças na regra de distribuição dos chamados royalties do petróleo, em que o governo perdeu receita por conta da divisão dos recursos dessa receita com outros estados. Outro fator que pode ter ocasionado essas mudanças, foi uma corrupção generalizada que se instalou nos governos do estado, em que diversos exgovernadores foram acusados de corrupção (GONÇALVES, 2019).

Outro fato de destaque na Tabela 1 é que, quanto às curtoses, somente o Estado do Paraná mostrou-se com uma curva leptocúrtica, mas por se tratar de dados que seguem uma distribuição não-normal, então não é possível afirmar que haja a presença de pontuações no orçamento geral. Cabe fazer a ressalva de que houve uma grande alta no orçamento por 
contada previdência, que quase triplicou de valor, e de que se antes compunha cerca de $10 \%$ do orçamento, passou a compor cerca de $24 \%$ do total do orçamento.

Ao analisar o orçamento das funções com limites mínimos constitucionais presentes na Tabela 2, é possível observar que também são apresentados valores não-normais em alguns estados. Em ambas as funções há estados que mostram uma distribuição nãonormal em suas variações, o que não indica sequer a presença do modelo incremental para alguns estados.

Tabela 2 | Análise das variações do orçamento por função de Despesa: saúde e educação

\begin{tabular}{|c|c|c|c|c|c|c|}
\hline \multirow[t]{2}{*}{ Estado } & \multicolumn{3}{|c|}{ Saúde } & \multicolumn{3}{|c|}{ Educação } \\
\hline & Teste & Valor-p & Curtose & Teste & Valor-p & Curtose \\
\hline$A C$ & 0,9788 & 0,9536 & 0,9971 & 0,9505 & 0,4982 & $-0,7838$ \\
\hline$A L$ & 0,8921 & 0,0601 & $-1,3977$ & 0,9244 & 0,1986 & 0,4412 \\
\hline AM & 0,9553 & 0,5781 & $-0,0009$ & 0,9566 & 0,6003 & $-0,7739$ \\
\hline AP & 0,9033 & 0,0909 & 2,6163 & 0,9438 & 0,3976 & 0,1370 \\
\hline BA & 0,8468 & $0,0122 *$ & 5,0543 & 0,8431 & $0,0108 *$ & 1,2340 \\
\hline CE & 0,9523 & 0,5277 & $-0,7580$ & 0,8298 & $0,0070 *$ & 5,3539 \\
\hline DF & 0,8918 & 0,0594 & 2,7177 & 0,8830 & $0,0433^{*}$ & 2,6002 \\
\hline ES & 0,9079 & 0,1077 & 3,3516 & 0,6485 & $0,0001 *$ & 10,8403 \\
\hline GO & 0,6425 & $0,0000^{*}$ & 10,5495 & 0,9158 & 0,1445 & $-0,6538$ \\
\hline MA & 0,9363 & 0,3060 & 1,6641 & 0,7417 & $0,0005^{*}$ & 7,8219 \\
\hline MG & 0,9481 & 0,4602 & 0,5170 & 0,9150 & 0,1401 & 2,1605 \\
\hline MS & 0,9567 & 0,6027 & $-0,8632$ & 0,9489 & 0,4725 & $-0,5057$ \\
\hline MT & 0,8317 & $0,0074 *$ & 2,3256 & 0,9608 & 0,6768 & 0,6368 \\
\hline PA & 0,9583 & 0,6301 & $-1,0211$ & 0,9676 & 0,7988 & 0,5009 \\
\hline PB & 0,9196 & 0,1665 & $-0,1421$ & 0,9778 & 0,9441 & 0,6345 \\
\hline PE & 0,9853 & 0,9920 & $-0,3277$ & 0,9572 & 0,6110 & $-0,7631$ \\
\hline PI & 0,7764 & $0,0014 *$ & 3,0736 & 0,7736 & $0,0012^{*}$ & 7,5995 \\
\hline PR & 0,9371 & 0,3152 & $-0,3545$ & 0,9193 & 0,1640 & 2,8263 \\
\hline
\end{tabular}




\begin{tabular}{|c|c|c|c|c|c|c|}
\hline RJ & 0,9486 & 0,4673 & $-1,0715$ & 0,9170 & 0,1509 & 1,4801 \\
\hline RN & 0,9488 & 0,4713 & 0,4649 & 0,9714 & 0,8602 & 1,1600 \\
\hline RO & 0,9360 & 0,3029 & 1,3131 & 0,9384 & 0,3293 & $-0,0247$ \\
\hline RR & 0,9614 & 0,6868 & 0,7059 & 0,9875 & 0,9968 & $-0,4939$ \\
\hline RS & 0,8473 & $0,0124 *$ & 2,5083 & 0,7631 & $0,0009 *$ & 6,8379 \\
\hline SC & 0,7856 & $0,0014^{*}$ & 6,9172 & 0,9600 & 0,6609 & 1,9357 \\
\hline SE & 0,9087 & 0,1108 & 0,8142 & 0,9538 & 0,5515 & $-0,8910$ \\
\hline SP & 0,8356 & $0,0084 *$ & 4,2900 & 0,9515 & 0,5137 & 1,9838 \\
\hline TO & 0,9763 & 0,9272 & 1,3400 & 0,9401 & 0,3501 & 1,1160 \\
\hline
\end{tabular}

Fonte: resultados da pesquisa * Nível de significância 5\%

Nos Estados da Bahia (BA), Goiás (GO) Mato Grosso (MT), Piauí (PI), Rio Grande do Sul (RS), Santa Catarina (SC) e São Paulo (SP) foram observados que as variações constituem uma distribuição não-normal, considerando o nível de significância de 5\%, na função saúde, o que configura a não aplicação do modelo incremental defendido por Wildavsky (1961), quando pensado em relação às funções orçamentárias. Importante salientar também que não configura o equilíbrio pontuado, já que não se atendeu a prerrogativa de estabilidade no orçamento, mesmo sendo possível observar que os graus de curtoses em alguns estados apresentaram valores altos, o que poderia ser considerado como uma pontuação no orçamento.

No caso da educação, além da Bahia, Piauí e Rio Grande do Sul, apresentam-se ainda com distribuições não-normais em suas variações o Ceará (CE), Distrito Federal (DF), Espírito Santo (ES) e o Maranhão (MA), mostrando mais uma vez que o modelo incremental também não se aplica nesse tipo de função em relação aos orçamentos estaduais. Também é possível notar que vários estados apresentaram altos graus de curtose, mas, por conta da distribuição de seus dados serem não-normais, não atendem a prerrogativa do incrementalismo, tampouco do equilíbrio pontuado.

Em relação à curtose é possível observar que somente um estado, na função saúde, apresentou normalidade em seus dados e com curva de curtose considerada leptocúrtica, que é o caso do ES, em que apresentou uma curtose com valor de 3,35, podendo concluir que existe a presença de pontuações no orçamento. Em relação à função educação, não 
houve nenhuma curtose com distribuição normal no orçamento e com curva leptocúrtica.

O Espírito Santo apresentou maiores pontuações no orçamento nas variações de $2004 / 2005$ e 2005/2006. Nesses anos ocorrem tanto variações positivas quanto negativas. De 2004 para 2005 a amplitude da variação é de cerca de 25\% positiva, isto é, o orçamento aumentou nesse ano. Já de 2005 para 2006 a amplitude é de cerca de 27\%, só que dessa vez negativa, fazendo com que o orçamento volte ao equilíbrio, caracterizando então o modelo do equilíbrio pontuado, em que há estabilidade seguida de grandes rupturas.

Contudo, é possível notar que 20 estados apresentaram distribuição normal na função saúde e também 20 estados mostraram uma distribuição normal para as variações de educação. Concluindo, portanto, que na maioria dos estados, nesses tipos de funções, o modelo incremental se aplica, ressalvado o caso do Espírito Santo, em que foi observada pontuação no orçamento.

Quanto às funções que não têm limites mínimos constitucionais, é possível observar padrões semelhantes àquelas que possuem o limite. Nota-se que na Tabela 3 foram apresentadas duas funções que não possuem a mesma tendência ou os mesmos pontos influenciadores para o aumento ou diminuição do gasto, ou seja, são totalmente independentes entre si.

Tabela 3 | Análise das variações do orçamento por função de Despesa: segurança pública e cultura

\begin{tabular}{|c|c|c|c|c|c|c|}
\hline Estado & \multicolumn{3}{|c|}{ Cultura } & \multicolumn{3}{c|}{ Segurança Pública } \\
\hline & Teste & Valor-p & Curtose & Teste & Valor-p & Curtose \\
\hline AC & 0,9711 & 0,8559 & $-0,2522$ & 0,9094 & 0,1137 & $-1,1874$ \\
\hline AL & 0,9461 & 0,4301 & $-0,9851$ & 0,9321 & 0,2635 & $-1,2435$ \\
\hline AM & 0,9252 & 0,2044 & 0,4154 & 0,9746 & 0,9063 & $-0,4184$ \\
\hline AP & 0,6310 & $0,0000 *$ & 10,6620 & 0,4170 & $0,0000^{*}$ & 14,8188 \\
\hline BA & 0,9567 & 0,6028 & 0,4859 & 0,9792 & 0,9696 & 0,6150 \\
\hline CE & 0,9447 & 0,4100 & 0,2722 & 0,8530 & $0,0151 *$ & 1,7690 \\
\hline DF & 0,9652 & 0,7565 & 1,0026 & 0,9567 & 0,6021 & 0,4325 \\
\hline ES & 0,8771 & $0,0350 *$ & 4,2628 & 0,8745 & $0,0319 *$ & 3,1861 \\
\hline
\end{tabular}




\begin{tabular}{|c|c|c|c|c|c|c|}
\hline GO & 0,7719 & $0,0012 *$ & 3,8896 & 0,9283 & 0,2294 & $-0,7803$ \\
\hline MA & 0,9715 & 0,8626 & $-0,6429$ & 0,8989 & 0,0773 & 0,5205 \\
\hline MG & 0,8937 & 0,0636 & $-1,0232$ & 0,8399 & $0,0097^{*}$ & 5,2802 \\
\hline MS & 0,9410 & 0,3617 & $-0,5880$ & 0,9420 & 0,3736 & 0,7824 \\
\hline MT & 0,7326 & $0,0004 *$ & 5,3465 & 0,8842 & $0,0451 *$ & 4,1789 \\
\hline PA & 0,8291 & $0,0068 *$ & 5,6525 & 0,8976 & 0,0737 & 2,0239 \\
\hline PB & 0,8241 & $0,0058 *$ & 3,4666 & 0,8819 & $0,0416^{*}$ & 2,8304 \\
\hline PE & 0,9254 & 0,2061 & $-0,0687$ & 0,9352 & 0,2941 & $-1,0955$ \\
\hline $\mathrm{PI}$ & 0,6403 & $0,0000 *$ & 10,5645 & 0,3054 & $0,0000 *$ & 15,9274 \\
\hline PR & 0,6644 & $0,0001 *$ & 10,4386 & 0,6101 & $0,0000^{*}$ & 10,7467 \\
\hline RJ & 0,9825 & 0,9802 & $-0,2253$ & 0,9117 & 0,1237 & 3,0653 \\
\hline $\mathrm{RN}$ & 0,9481 & 0,4595 & $-0,1624$ & 0,9668 & 0,7840 & $-0,3049$ \\
\hline RO & 0,7827 & $0,0016^{*}$ & 0,5482 & 0,8973 & 0,0726 & 3,6440 \\
\hline $\mathrm{RR}$ & 0,9245 & 0,1991 & 2,2129 & 0,9588 & 0,6404 & $-0,7273$ \\
\hline RS & 0,9634 & 0,7242 & $-0,6421$ & 0,9108 & 0,1197 & 1,9880 \\
\hline SC & 0,5267 & $0,0000 *$ & 13,4273 & 0,3796 & $0,0000^{*}$ & 15,3002 \\
\hline SE & 0,8520 & $0,0146 *$ & 1,3856 & 0,8848 & $0,0461 *$ & 0,7891 \\
\hline SP & 0,9750 & 0,9119 & $-0,5608$ & 0,8419 & $0,0104^{*}$ & 4,1039 \\
\hline TO & 0,9108 & 0,1200 & 1,0892 & 0,9698 & 0,8354 & $-0,3373$ \\
\hline
\end{tabular}

Fonte: resultados da pesquisa *Nível de significância 5\%

Observa-se na Tabela 3 que, tanto na função cultura quanto na função segurança pública, são apresentados diversos estados com distribuição não-normal, 11 estados em cada uma das funções. Nota-se, portanto, a ausência do modelo incremental para essas funções nos respectivos estados, ou mesmo ao modelo do Equilíbrio Pontuado, justamente pela falta de pontuações e um orçamento que não segue padrões semelhantes ao incrementalismo.

É importante salientar que, apesar da Tabela 3 mostrar um número considerável de estados que seguem uma distribuição não-normal, também são apresentados 16 estados que apresentam a distribuição normal. Ressalta-se o fato de que no caso do Rio de Janeiro, na área de segurança pública, é possível observar que os dados seguem uma 
distribuição normal e que o nível encontrado para a medida de curtose configura uma curva leptocúrtica, já que apresenta um valor maior que 3, concluindo-se que o orçamento sofre incrementos, mas que acontece uma ruptura em algum momento.

As pontuações no orçamento de segurança pública do Rio de Janeiro ocorrem no período que aconteceu o evento esportivo dos Jogos Pan-Americano, no ano de 2007. Verificou-se que nos anos de 2007/2008 houve uma amplitude de variação negativa acima de 33\%, e isso pode ter acontecido devido ao evento, em que até o ano de 2007 o governo precisava investir mais nessa área para garantir a segurança dos espectadores dos jogos, já que se trata de um evento que movimenta o turismo, tanto interno quanto externo, além de ser um evento que leva a imagem do país para o resto do mundo. Já entre 2008/2009 a variação voltou a ser positiva, para atingir o nível considerado normal para aquela área, então voltando para o período de equilíbrio.

Outro estado em que foram apresentadas pontuações no orçamento foi Rondônia (RO), em que houve mudanças no orçamento relativas à área de segurança pública. $A$ primeira variação encontrada ocorreu entre os anos de 2007 e 2008, em que houve alterações positivas acima de 20\%. Lessa (2018) afirma que o Plano Estadual de Segurança Pública foi expirado em 2007 e que este não foi substituído, o que deixa essa área sem rumo. Já a segunda variação foi entre os anos de 2015 e 2016, tendo variação negativa de cerca de $25 \%$, o que pode ser explicado pelo momento em que o país vivia em meio ao processo de impeachment da Presidente da República e que pode ter afetado os orçamentos.

Verifica-se ainda na Tabela 3 que alguns Estados, como Amapá (AP), Santa Catarina e Piauí apresentam altos graus de curtose, mas estes não podem ser interpretados como pontuações no orçamento devido à ausência da distribuição normal das variações das funções. Mas um fator que é importante salientar é a falta de padrão que existe nos orçamentos dos estados brasileiros, o que faz com que não seja possível a identificação sequer da teoria incremental.

As variações encontradas nos orçamentos se devem ao contexto político-econômico em que o país está inserido. No ano de 2015, a crise econômica agravada e a redução das receitas federais fizeram com que o Governo Federal tomasse decisões de realizar cortes 
orçamentários nas mais diversas áreas (CUNHA et al. 2015), incluído saúde e educação e também cultura e segurança pública.

\section{Conclusão}

Esta pesquisa teve como objetivo identificar a aderência dos orçamentos dos governos estaduais à teoria do equilíbrio pontuado, com a finalidade de verificar se os orçamentos de fato são incrementais ou se ocorrem pontuações neles. Observou-se que, no caso do orçamento total, esses de fato seguem uma distribuição normal, indicando na maioria dos casos se tratarem de orçamentos em modelo incremental. A exceção se faz para dois estados, o Rio de Janeiro e o Paraná, onde ambos seguiram uma distribuição não-normal em seus dados, o que vai de encontro ao que a teoria incremental afirma.

Testou-se ainda a normalidade e a curtose para quatro funções orçamentárias, duas com mínimos constitucionais definidos e duas sem limites definidos. Notou-se que na maioria dos casos das funções com mínimos constitucionais, as distribuições são normais, indicando que estas seguem o padrão incremental, observando-se também que, nos casos que houve normalidade de dados, somente um estado mostrou-se com pontuações. Foi o caso do Espírito Santo, que apresentou curva leptocúrtica na função saúde, atestando a teoria do equilíbrio pontuado nesse estado.

Quanto às funções sem mínimos constitucionais definidos, estas se mostraram em acerca de onze estados com distribuições não-normais. Ou seja, mostraram-se com comportamento completamente diferente de um modelo incremental. Um fato que foi identificado é que no caso do Rio de Janeiro mostrou-se uma distribuição normal na função segurança pública e a sua curtose pôde ser definida como uma curva leptocúrtica, confirmando a teoria do equilíbrio pontuado. Deve-se atentar ainda para o fato de ter acontecido um evento que gera turismo no estado e que, portanto, havia uma necessidade de o governo investir mais fortemente nessa função para garantir a segurança dos espectadores do evento.

Por fim, em relação à hipótese levantada no início do trabalho, de que os orçamentos seguem pequenos incrementos e são pontualmente alterados por grandes variações, não 
se aplica em sua totalidade para os estados brasileiros, entretanto nota-se com frequência a presença do modelo incremental nas funções analisadas. Como sugestão para pesquisas futuras, fica a ideia de se fazer a ampliação da análise para outras funções de despesas, compreendendo um período maior de análise e talvez analisar outro estágio despesa, como a liquidação ou pagamento. Uma outra sugestão de pesquisa é verificar se as pontuações no orçamento têm relação com os ciclos políticos.

\section{Referências bibliográficas}

BARCELOS, Carlos Leonardo Klein. Governança orçamentária e mudança institucional: o caso da norma geral de direito financeiro - Lei $n^{\circ}$ 4.320/64. 2012. xi, 276 f., il. Tese (Doutorado em Administração) Universidade de Brasília, Brasília, 2012. Disponível em: https://repositorio.unb.br/ handle/10482/12572 Acesso em 14 jan. 2019

BAUMgARTNER, Frank R.; Jones, Bryan D. Agenda dynamics and policy subsystems. The journal of Politics, v. 53, n. 4, p. 1044-1074, 1991. DOI: https://doi.org/10.2307/2131866 Disponível em: https://www.journals.uchicago.edu/doi/abs/10.2307/2131866 Acesso em 01 out. 2019

BRASIL, Felipe; CAPELLA, Ana Cláudia. A dinâmica da atenção governamental sobre as políticas de saúde no Brasil: equilíbrio e pontuações nas primeiras décadas pós-redemocratização (1986-2003). Saúde e Sociedade, v. 28, p. 80-96, 2019. DOI: https://doi.org/10.1590/ s0104-12902019180570 Disponível em: http://www.scielo.br/scielo.php?pid=S010412902019000300080\&script=sci_arttext\&tlng=pt Acesso em 14 mar. 2019

BReUnIG, Christian; KoskI, Chris. Punctuated equilibria and budgets in the American states. Policy Studies Journal, v. 34, n. 3, p. 363-379, 2006. DOI: https://doi.org/10.1111/j.15410072.2006.00177.x Disponível em: https://onlinelibrary.wiley.com/doi/full/10.1111/j.15410072.2006.00177.x? Acesso em 15 jul. 2019

Carvalho, Daniel Izaias de. Análise da execução orçamentária do Distrito Federal entre 2000 e 2015, sob a perspectiva da Teoria do Equilíbrio Pontuado. 2017. x, 86 f., il. Dissertação (Mestrado em Administração) Universidade de Brasília, Brasília, 2017. Disponível em: https://repositorio. unb.br/handle/10482/23300 Acesso em 20 jan. 2020

CARVAlHo, Daniel Izaias de. Teoria do Equilíbrio Pontuado: uma análise da execução orçamentária no Brasil no período de 1980-2014. Revista do Serviço Público, v. 69, n. 1, p. 85-110, 2018. DOI: https://doi.org/10.21874/rsp.v69i1.1639 Disponível em: https://revista.enap.gov.br/index.php/ RSP/article/view/1639 Acesso em 15 jul. 2019

Davis, Otto A.; DeMPSTER, Michael Alan Howarth; WiLdAVSkY, Aaron. A theory of the budgetary process. American Political Science Review, v. 60, n. 3, p. 529-547, 1966. DOI: https://doi. 
org/10.2307/1952969 Disponível em: https://www.cambridge.org/core/journals/americanpolitical-science-review/article/theory-of-the-budgetary-process/03E867D70A5B952EC083092E8 410ECE2 Acesso em 13 mar. 2020

DOESER, Fredrik; EIDENFALK, Joakim. The importance of windows of opportunity for foreign policy change. International area studies review, v. 16, n. 4, p. 390-406, 2013. DOI: https:// doi.org/10.1177/2233865913512117 Disponível em: https://journals.sagepub.com/doi/ abs/10.1177/2233865913512117 Acesso em 22 fev. 2020

FitTIPALDI, I.; COSTA, S.; ARAújo, C. O gasto público federal brasileiro: um perfil incrementalista?. Revista do Serviço Público, v. 68, n. 3, p. 611-630, 29 set. 2017. DOI: https://doi.org/10.21874/rsp. v68i3.1332 Disponível em: https://revista.enap.gov.br/index.php/RSP/article/view/1332 Acesso em 12 fev. 2020

GıACOMONI, James. Orçamento público. rev. e atual. São Paulo: Atlas, 2017

GonçALVES, Rafael Soares. As eleições de 2018 no Estado de Rio de Janeiro: crônicas de um desastre político. IdeAs. Idées d'Amériques, n. 13, 2019. DOI: https://doi.org/10.4000/ideas.5443 Disponível em: https://journals.openedition.org/ideas/5443\#quotation Acesso em 14 mar. 2019

Jones, Bryan D.; BAUMgartner, Frank R. A model of choice for public policy. Journal of Public Administration Research and Theory, v. 15, n. 3, p. 325-351, 2005. DOI: https://doi.org/10.1093/ jopart/mui018 Disponível em: https://academic.oup.com/jpart/article-abstract/15/3/325/941117 Acesso em 13 mar. 2020

JORDAN, Meagan M. Punctuations and agendas: a new look at local government budget expenditures. Journal of Policy Analysis and Management, v. 22, n. 3, p. 345-360, 2003. DOI: https://doi.org/10.1002/pam.10136 Disponível em: https://onlinelibrary.wiley.com/doi/ abs/10.1002/pam.10136? Acesso em 18 fev. 2020

LESSA, Sávio Antiógenes Borges. Planejamento estratégico e política de segurança pública: análise do processo de formulação das políticas de segurança pública implementadas pelo estado de Rondônia no período de 2008 a 2017. 2018. Disponível em: https://www.lume.ufrgs.br/ handle/10183/193537 Acesso em: 23 ago. 2020

LINDBLOM, Charles E. The science of" muddling through". Public administration review, p. 79-88, 1959. DOI: $10.2307 / 973677$ Disponível em: https://www.jstor.org/stable/973677?seq=1 Acesso em 10 jan. 2020

Melo, V. S. et al. Tomada de decisão em orçamento: o caso da Universidade Federal de Santa Catarina. Revista Gual, Florianópolis, v. 11, n. 1, p. 253-275, 2018 DOI: Disponível em: https:// doi.org/10.5007/1983-4535.2018v11n1p253 https://periodicos.ufsc.br/index.php/gual/article/ view/1983-4535.2018v11n1p253 Acesso em 15 jul. 2019

Metten, Antoine et al. A introdução do complexo econômico industrial da saúde na agenda de desenvolvimento: uma análise a partir do modelo de fluxos múltiplos de Kingdon. Revista de Administração Pública, Rio de Janeiro, v. 49, n. 4, p. 915 a 936, jun. 2015. ISSN 1982-3134. DOI: http://dx.doi.org/10.1590/0034-7612123873 Disponível em: http://bibliotecadigital.fgv.br/ojs/ index.php/rap/article/view/51568 Acesso em: 21 ago. 2020 
Mortensen, Peter B. Policy punctuations in Danish local budgeting. Public Administration, v. 83, n. 4, p. 931-950, 2005. DOI: https://doi.org/10.1111/j.0033-3298.2005.00484.x Disponível em: https://onlinelibrary.wiley.com/doi/full/10.1111/j.0033-3298.2005.00484.x? Acesso em 11 fev. 2020

NASCIMENTO, Sidnei Pereira do. Guerra fiscal: uma avaliação comparativa entre alguns estados participantes. Economia aplicada, v. 12, n. 4, p. 677-706, 2008. DOI: https://doi.org/10.1590/ S1413-80502008000400007 Disponível em: https://www.scielo.br/pdf/ecoa/v12n4/07.pdf Acesso em 25 fev. 2020

Nowlin, Matthew C. Theories of the policy process: state of the research and emerging trends. Policy Studies Journal, v. 39, p. 41-60, 2011. DOI: https://doi.org/10.1111/j.15410072.2010.00389_4.x Disponível em: https://onlinelibrary.wiley.com/doi/full/10.1111/j.15410072.2010.00389_4.x? Acesso em 13 mar. 2020

Nugent, Neill; SAURugger, Sabine. Organizational structuring: the case of the European Commission and its external policy responsibilities. Journal of European Public Policy, v. 9, n. 3, p. 345-364, 2002. DOI: https://doi.org/10.1080/13501760210138787 Disponível em: https://www. tandfonline.com/doi/abs/10.1080/13501760210138787 Acesso em 21 ago. 2020

OllaIK, Leila Giandoni; Wenceslau, Juliana; SerPA, Selma Maria Hayakawa Cunha; MedeIRos, Janann Joslin. Novas direções para pesquisas orçamentárias no Brasil. Revista de Políticas Públicas, v. 15, n. 2, p. 347-357, 2012. Disponível em: http://www.periodicoseletronicos.ufma.br/ index.php/rppublica/article/view/850/873 Acesso em 15 mar. 2020

PADGETT, John F. Managing garbage can hierarchies. Administrative Science Quarterly, p. 583-604, 1980. DOI: 10.2307/2392282 Disponível em: https://www.jstor.org/stable/2392282 Acesso em 21 fev. 2020

PORSSE, Alexandre A; HADDAD, Eduardo Amaral; RIBEIRO, Eduardo Pontual. Competição tributária regional no Brasil: análise com um modelo EGC inter-regional. Pesquisa e Planejamento Econômico, v. 38, n. 3, 2008. Disponível em: https://ppe.ipea.gov.br/index.php/ppe/article/ viewFile/1185/1036 Acesso em: 25 ago. 2020

RIDDE, Valéry. Policy implementation in an African state: an extension of Kingdon's MultipleStreams Approach. Public Administration, v. 87, n. 4, p. 938-954, 2009. DOI: https://doi. org/10.1111/j.1467-9299.2009.01792.x Disponível em: https://onlinelibrary.wiley.com/doi/ abs/10.1111/j.1467-9299.2009.01792.x Acesso em 21 ago. 2020

SCHICK, Allen. O PPB e o orçamento incremental. Revista de Administração Pública, v. 10, n. 2, p. 65-84, 1976. Disponível em: http://bibliotecadigital.fgv.br/ojs/index.php/rap/article/ download/6086/4714 Acesso em 12 abr. 2019

Silvestre, Hugo Consciência; Araúso, Joaquim Filipe Ferraz Esteves de. Teoria do equilíbrio pontuado nas políticas públicas brasileiras: o caso do Ceará. Revista de Administração Contemporânea, v. 19, n. 6, p. 696-711, 2015. DOI: http://dx.doi.org/10.1590/19827849rac20151721 Disponível em: http://www.scielo.br/scielo.php?pid=S141565552015000600003\&script=sci_arttext Acesso em 15 jul. 2019 
TIEBout, Charles M. A pure theory of local expenditures. Journal of political economy, v. 64, n. 5, p. 416-424, 1956. Disponível em: https://www.jstor.org/stable/pdf/1826343.pdf Acesso em 25 ago. 2020

TRAVIS, Rick; ZAHARIADIS, Nikolaos. A multiple streams model of US foreign aid policy. Policy Studies Journal, v. 30, n. 4, p. 495-514, 2002. DOI: https://doi.org/10.1111/j.1541-0072.2002. tb02160.x Disponível em: https://onlinelibrary.wiley.com/doi/abs/10.1111/j.1541-0072.2002. tb02160.x Acesso em 21 ago. 2020

WANAT, John. Bases of budgetary incrementalism. American Political Science Review, v. 68, n. 3, p. 1221-1228, 1974. DOI: 10.2307/1959158 Disponível em: https://www.cambridge.org/core/ journals/american-political-science-review/article/bases-of-budgetary-incrementalism/0C645313 849BAB2CFABE7C618912C7F2 Acesso em 20 ago. 2020

WILDAVSKY, Aaron. Political implications of budgetary reform. Public administration review, p. 183190, 1961. DOI: 10.2307/973628 Disponível em: https://www.jstor.org/stable/973628 Acesso em 10 fev. 2020

WILLIAMSON, Oliver E. A rational theory of the federal budgeting process. Papers on Non-Market Decision Making, v. 2, n. 1, p. 71-89, 1967. DOI: 10.1007/BF01718653 Disponível em: https://link. springer.com/article/10.1007/BF01718653 Acesso em 20 ago. 2020

\section{Marlon Coqueiro Galdino \\ (iD) ORCID: 0000-0002-5169-1601}

Mestre em Contabilidade Financeira pela Faculdade de Ciências Contábeis da Universidade Federal de Uberlândia (2020). Possui graduação em Ciências Contábeis pela Universidade Estadual do Sudoeste da Bahia (2015).

E-mail: Marlon-galdino@hotmail.com

\section{Maria Elisabeth Moreira Carvalho Andrade}

ORCID: 0000-0002-4176-9153

Professora Adjunto da Universidade Federal de Uberlândia - UFU. Doutora em Ciências pela Faculdade de Economia, Administração e Contabilidade de São Paulo - FEA/USP (2015). Mestre em Ciências: área Controladoria e Contabilidade (2010) pela Faculdade de Economia, Administração e Contabilidade de Ribeirão Preto da Universidade de São Paulo. Atua no curso de Ciências Contábeis (graduação e pós-graduação).

E-mail: maria.elisabeth@ufu.br 